\title{
Giant right atrial hemangioma: Correlation between magnetic resonance imaging and histopathologic evaluation
}

\author{
Cristina Sánchez-Enrique, MD, ${ }^{\mathrm{a}}$ David Vivas, $\mathrm{MD}, \mathrm{PhD},{ }^{\mathrm{a}}$ Manuel Carnero Alcázar, $\mathrm{MD}, \mathrm{PhD},{ }^{\mathrm{b}}$ and \\ Isidre Vilacosta, $\mathrm{MD}, \mathrm{PhD},{ }^{\mathrm{a}}$ Madrid, Spain
}

\author{
From the Departments of ${ }^{\mathrm{a} C a r d i o l o g y}$ and ${ }^{\mathrm{b}}$ Cardiac Surgery, Hospital Clínico San Carlos, Madrid, Spain. \\ Disclosures: Authors have nothing to disclose with regard to commercial support. \\ Received for publication June 10, 2015; accepted for publication June 13, 2015; available ahead of print July 18 , \\ 2015 . \\ Address for reprints: Cristina Sánchez-Enrique, MD, Department of Cardiology, Hospital Clínico San Carlos, \\ Profesor Martín Lagos s/n, 28040 Madrid, Spain (E-mail: cristinasess@ gmail.com). \\ J Thorac Cardiovasc Surg 2015;150:e51-3 \\ $0022-5223 / \$ 36.00$ \\ Copyright (C) 2015 by The American Association for Thoracic Surgery \\ http://dx.doi.org/10.1016/j.jtcvs.2015.06.034
}

Cardiac hemangioma is an uncommon benign tumor most frequently located in the epicardium but also described within the myocardium and endocardium. ${ }^{1}$ We report a rare case of a giant right atrial mixed hemangioma and describe the correlation among echocardiographic, magnetic resonance, intraoperative, and histologic imaging.

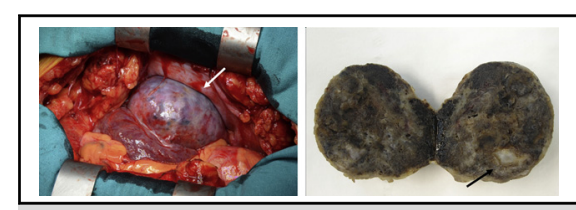

Blue right atrial mass corresponding to a giant hemangioma.

Central Message

Correlations are described among echocardiographic, magnetic resonance, intraoperative, and histologic imaging of a giant right atrial mixed hemangioma.

See Editorial Commentary page e54.

\section{CLINICAL SUMMARY}

A symptom-free 74-year-old man was referred to our hospital with an incidentally found huge right atrial mass. On physical examination, significant findings included normal blood pressure, lack of jugular venous distention, normal carotid and peripheral pulses, and clear lung fields. On auscultation, $S_{1}$ and $S_{2}$ were normal, and no extra heart sounds were heard. The electrocardiogram exhibited a normal sinus rhythm with left axis deviation. Chest radiography revealed cardiomegaly. A transthoracic echocardiogram showed a large, rounded, echo-bright mass containing hypoechoic areas (Figure 1, A$C$ ). The mass was located within the right atrium and attached to the lateral wall, with no hemodynamic obstruction.

Cardiac magnetic resonance imaging was requested for tissue characterization. This technique demonstrated a huge $(55 \times 50 \mathrm{~mm})$, well-circumscribed mass with a broad base of attachment arising from the right atrial posterior wall up to the superior vena cava. The lesion had intermediate signal intensity on T1-weighted images and was hyperintense on T2-weighted images (Figure 1,D). A heterogeneous hyperintense enhancement immediately after gadolinium injection demonstrated a high vascularized mass (Figure 1,E). Coronary angiography showed a rich vascular tumor blush that originated from the right coronary artery (Figure 1, F).

At surgery, an enormous $(6 \times 6 \mathrm{~cm})$ tumor was completely resected (Figure 2, A), and its feeding vessel coming from the right coronary artery was ligated. In addition, a portion of the right atrial wall was removed and replaced with autologous pericardium (Figure 2, B).

Macroscopic evaluation showed a reddish blue encapsulated tumor with an irregular surface (Figure 2, C). In a cross- section, white and yellow areas of degeneration and calci-fication, as well as some bleeding regions, were observed (Figure 2, D). Histologic examination demonstrated multiple, thin-walled vessels and focal calcifications. A few tissue areas with intercommunicating dysplastic arterial and venous structures and smaller endothelium-lined spaces separated by connective tissue were also observed (Figure 2, $E-H$ ). The final diagnosis was a mixed capillary, cavernous, and arteriovenous hemangioma. The patient's postoperative course was uneventful.

\section{DISCUSSION}

Cardiac hemangioma is an uncommon benign tumor (accounting for $5 \%$ of all benign cardiac tumors). Cardiac hemangiomas are most frequently located in the epicardium but have also been described within the myocardium and endocardium. ${ }^{1}$ Right atrial hemangiomas are extremely rare. ${ }^{2}$ Clinical manifestations vary according to the size and location of the tumor. In the case presented here, the patient had no symptoms despite the tumor's huge size. Typically, these tumors are hyperechoic on echocardiography, but magnetic resonance imaging is the imaging technique that precisely characterizes the vascular nature of the tumor. On T1-weighted imaging, the mass is seen as isointense or hyperintense as a result of slow blood flow, whereas it is always hyperintense in T2-weighted images. After gadolinium administration, there is a marked enhancement that may be heterogeneous if there are degenerative areas, as occurred in our case. ${ }^{3}$ Differential diagnosis includes myxomas, the most frequent primary cardiac tumor, in which first-pass enhancement is usually mild or not apparent. 


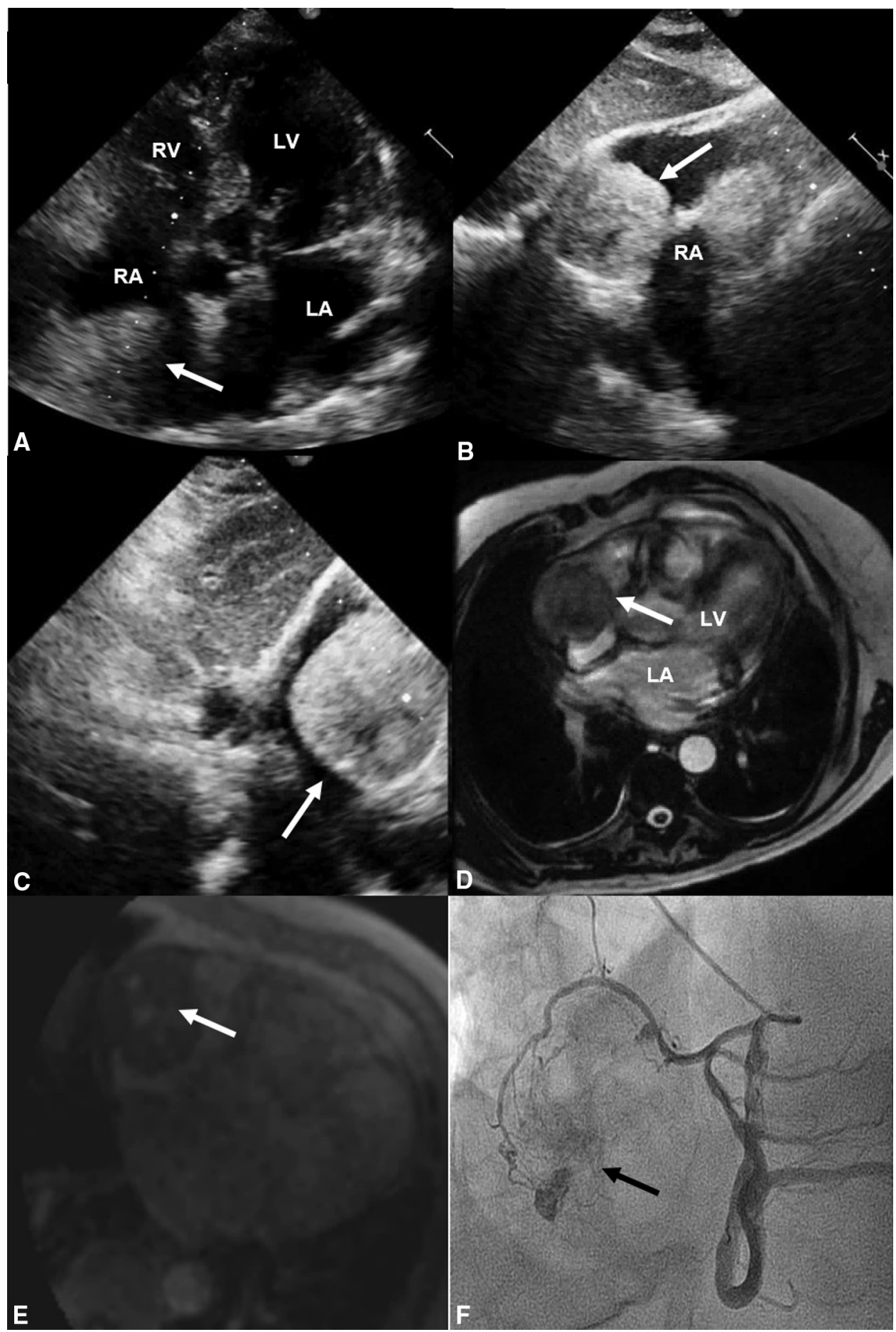

FIGURE 1. A-C, Two-dimensional echocardiography. A, An apical 4-chamber view reveals a well-circumscribed, circular hyperechoic mass (arrow) located in the right atrium. B and C, Subcostal views demonstrate hypoechoic areas within the mass (arrows). D, A 4-chamber cine steady-state free precession cardiac magnetic resonance imaging frame demonstrates a hypointense spherical mass within the right atrium (arrow). E, A perfusion sequence of cardiac magnetic resonance imaging shows areas of avid enhancement of the tumor. F, A coronary angiogram shows the right coronary artery feeding the tumor and delineates a tumor blush (arrows). $R V$, Right ventricle; $L V$, left ventricle; $R A$, right atrium; $L A$, left atrium.

Histologically, hemangiomas are characterized by proliferation of blood vessel endothelial cells and can be classified into 3 types: cavernous, characterized by dilated, thin- walled vessels; capillary, composed of smaller, capillarylike vessels; and arteriovenous, in which dysplastic arteries and veins are found. ${ }^{4}$ In our patient, a mixture combining 


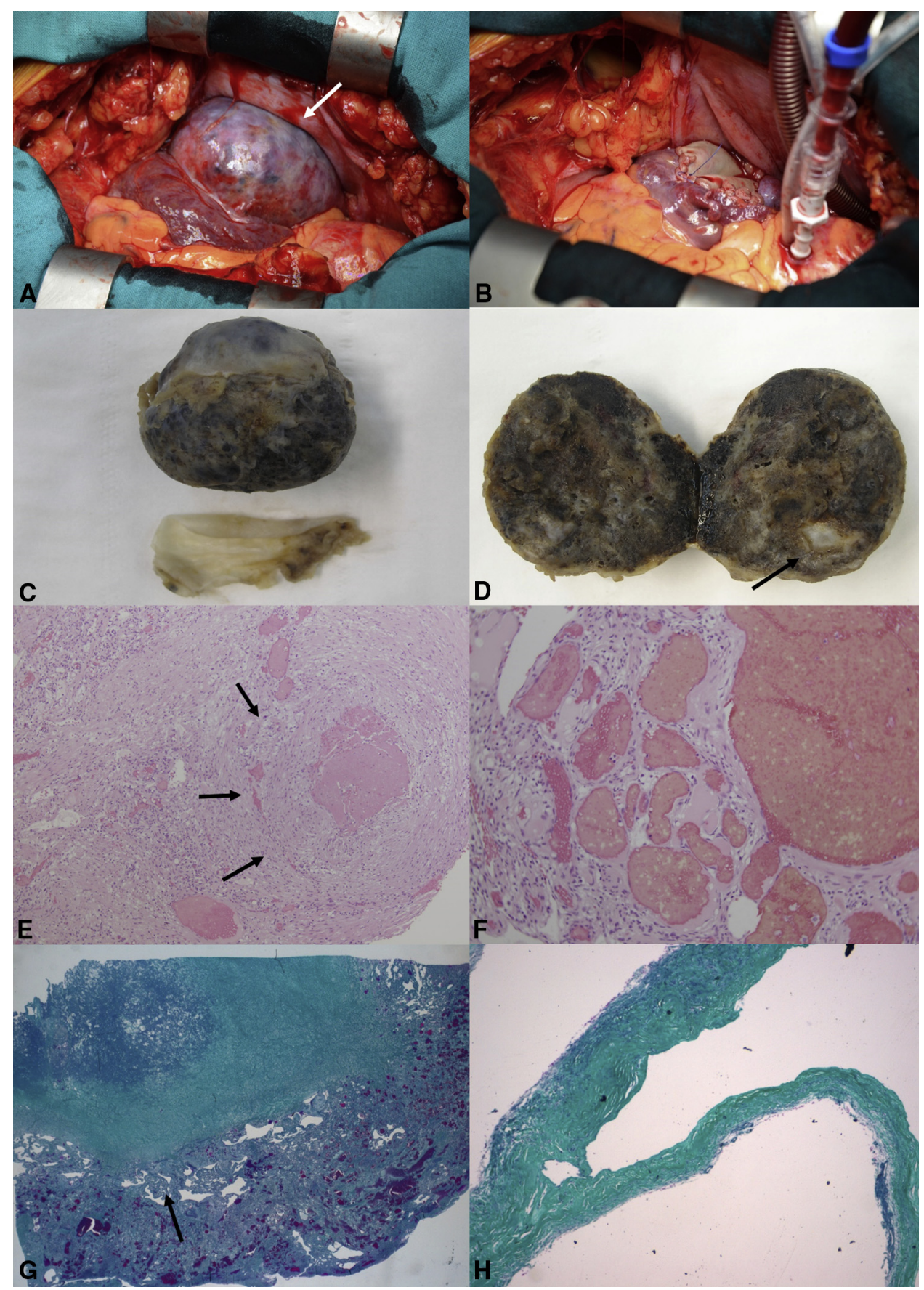

FIGURE 2. A, Intraoperative image demonstrates a blue right atrial mass (arrow). B, Intraoperative image demonstrates a pericardium patch closing the right atrium wall. C, Photograph shows the resected tumor with a capsule. D, Cross-sectioning reveals a spongy quality and white areas of degeneration. E and F, Histologic examination shows small thick- and thin-walled vessels with cavernous spaces (hematoxylin and eosin stain). G and H, Masson trichrome stain reveals fibrous areas and demonstrates the fibrous origin of the capsule.

the features of all 3 histologic categories was seen. Because the natural history of cardiac hemangioma is unpredictable, surgery is often indicated.

\section{References}

1. Shapiro LM. Cardiac tumours: diagnosis and management. Heart. 2001;85: 218-22.
2. Baird C, Blalock S, Bengur R, Ikemba C. Right atrial hemangioma in the newborn: utility of fetal imaging. Ann Pediatr Cardiol. 2012;5:81-4.

3. Randhawa K, Ganeshan A, Hoey ET. Magnetic resonance imaging of cardiac tumors: part 1, sequences, protocols, and benign tumors. Curr Probl Diagn Radiol. 2011;40:158-68.

4. Ainsworth CD, Salehian O, Nair V, Whitlock RP. A bloody mass: rare cardiac tumor as a cause of symptomatic ventricular arrhythmias. Circulation. 2012;126: 1923-31. 